\title{
Imaging Of Cellular Trafficking In Skin Using Multiphoton And Handheld Confocal Microscopy Techniques.
}

\author{
Simon C. Watkins, Glenn D. Papworth \\ Center for Biologic Imaging, University of Pittsburgh, Pittsburgh PA, 15261.
}

We will present data showing the utility of both multiphoton microscopy and handheld confocal microscopy techniques for minimally invasive investigation of cell trafficking in human and murine skin.

One alternative to the conventional cytotoxic agents used in conventional cancer threrapies is a more defined molecular approach for cancer immune treatment; promotion of the immune system specifically to target and eliminate tumor cells on the basis of expression of tumor-associated antigens (TAA). It has been well documented that certain antigen-presenting cells (APC) are capable of recognizing, processing and presenting TAA, in turn initiating a specific antitumor immune response (1). Results from several laboratories and clinical trials $(2,3)$ suggested significant efficacy of TAA-pulsed APC to generate a more efficient and effective anti-tumor immune response, following administration to tumor-bearing hosts.

It is fundamentally necessary to dynamically assess cell abundance within the microenvironment of the tumor in the presence of the appropriate therapeutic agent. Multiphoton microscopy and hand-held confocal microscopy were used to assess the trafficking of antigen presenting cells in mice, both in vivo and ex vivo. These imaging techniques are particularly suited to such investigations, because of their deep-tissue and minimally-invasive cellular imaging capabilities.

1. Shurin MR. Dendritic cells presenting tumor antigen. Cancer Immunol Immunother 1996;43(3):158-64.

2. Hamblin TJ. From Dendritic cells to tumor vaccines. Lancet 1996;347:705-706.

3. Girolomoni G, Ricciardi-Castagnoli P. Dendritic cells hold promise for immunotherapy. Immunol. Today 1997;18:1021-1024. 

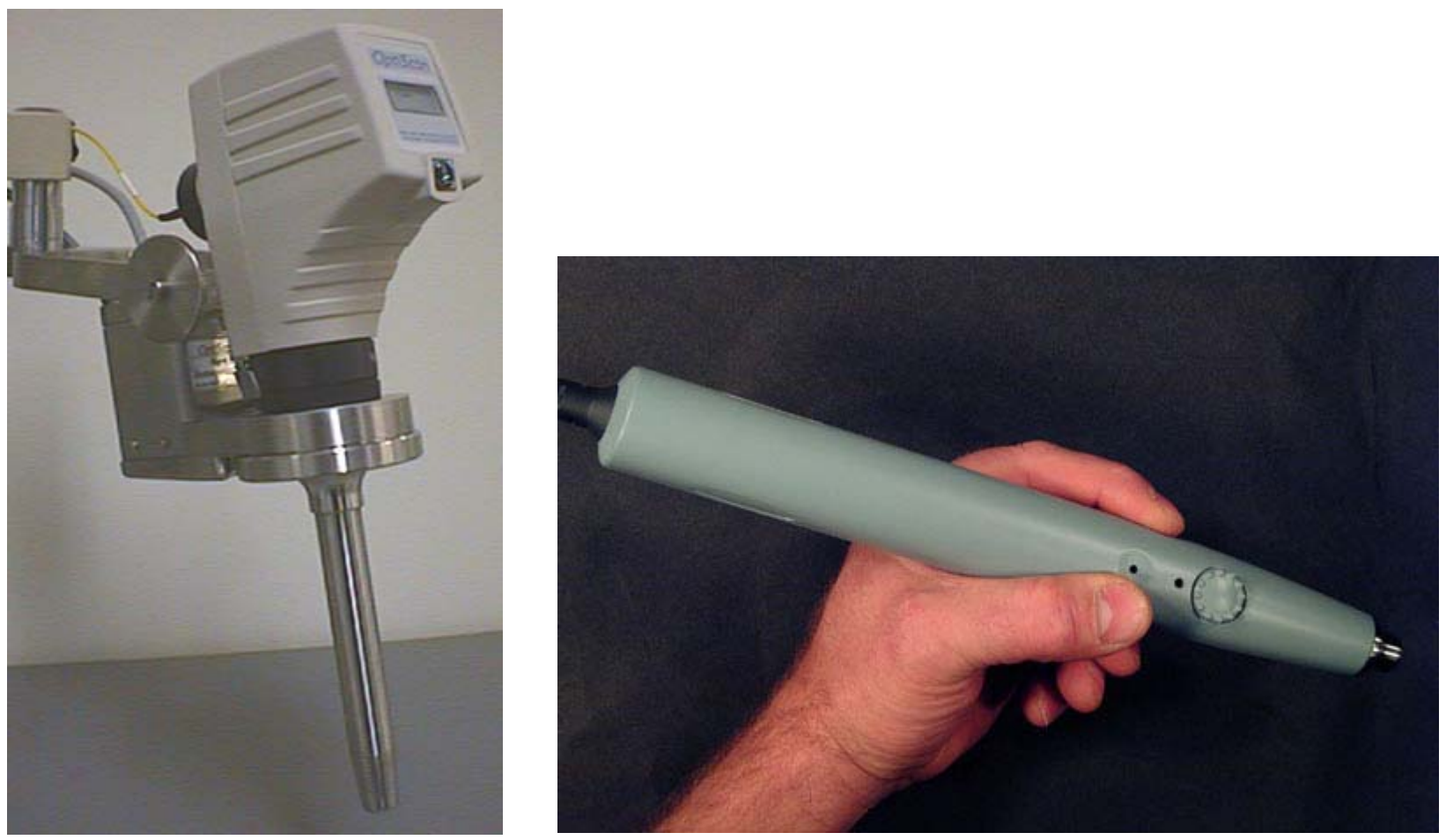

FIG. 1. Hand-held confocal imaging probes

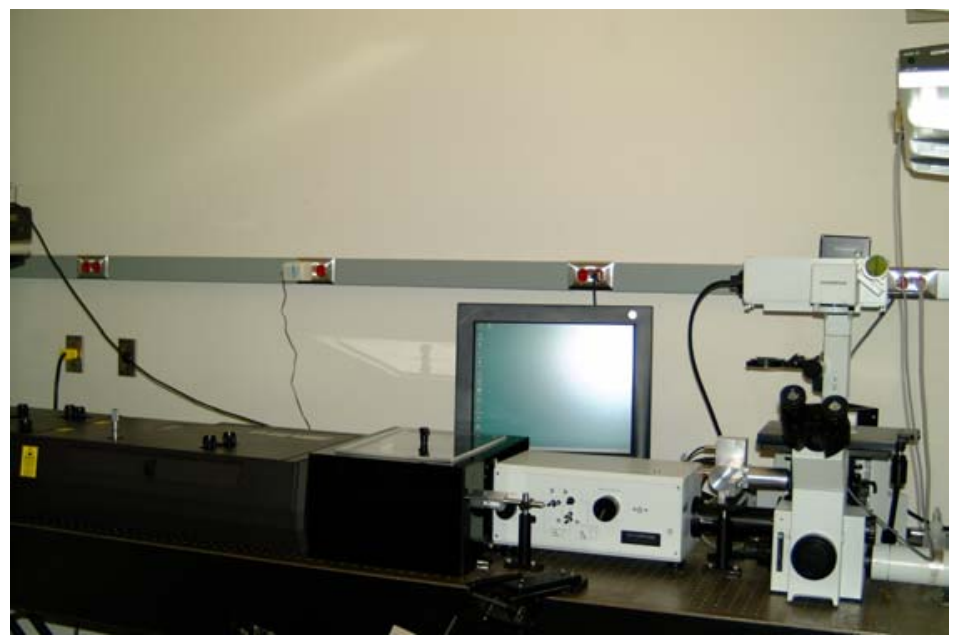

FIG.2. Multiphoton microscope system 